\title{
Acute Effect of Coconut Oil Consumption does not Affect Postprandial Human Cytokines in Healthy Overweight Women
}

\author{
Daniela Mayumi Usuda Prado Rocha ${ }^{1 *}$, Ana Paula Silva Caldas ${ }^{1}$, Olívia Gonçalves Leão Coelho ${ }^{1}$, Ana \\ Luiza Gomes Domingos ${ }^{1}$, Laís Monteiro Rodrigues Loureiro ${ }^{1}$, Leandro Licursi de Oliveira ${ }^{2}$, Josefina \\ Bressan $^{1}$
}

${ }^{1}$ Federal University of Vicosa, Department of Nutrition and Health, Viçosa, Minas Gerais, Brazil ${ }^{2}$ Federal University of Vicosa, Department of General Biology, Viçosa, Minas Gerais, Brazil

*Corresponding author: Daniela Mayumi Usuda Prado Rocha, Av PH Rolfs s/n, Federal University of Vicosa, Department of Nutrition and Health, Viçosa, Minas Gerais, CEP: 36570-900, Brazil, Tel: +55 31 3899 3388; E-mail: mayumi.dani@gmail.com

\begin{abstract}
The coconut oil is popularly recognized for its anti-inflammatory properties, but research has shown conflicting results. Thus, our aim was to assess the postprandial inflammatory response of moderate coconut oil intake. We evaluated the acute consumption of a breakfast, offered in a single day, containing $25 \mathrm{~mL}$ of coconut oil (test) or $25 \mathrm{~mL}$ of olive oil (control)on postprandial serum inflammatory cytokines (IL-2, IL-4, IL-6, IL-10, IL-17A, IFN- $\gamma$ and TNF) in healthy overweight women. No change was observed in relation to the effect of fatty meals, the time and the interaction of both factors (meal x time). Thus, despite being a saturated fat source, coconut oil consumption did not promote an inflammatory response. Further studies are needed considering a larger intervention period to check long-term effect.
\end{abstract}

Keywords: Inflammation; Overweight; interleukin; Fatty acids; Coconut fat
Received Date: October 08, 2016

Accepted Date: October 22, 2016

Published Date: October 28, 2016

Citation: Usuda Prado Rocha, D.M., et al. Acute Effect of Coconut Oil Consumption does not Affect Postprandial Human Cytokines in Healthy Overweight Women. (2016) Int J Food Nutr Sci 3(2): 354-358.

DOI: $10.15436 / 2377-0619.16 .1160$

\section{Introduction}

There is a great demand for weight loss strategies and reducing body fat, especially for overweight women, increasingly popularized by media, which has invested in marketing of food with "miracle" potential, and more recently highlighting the coconut oil as "miraculous" fat ${ }^{[1]}$. Coconut oil contains high proportions of Medium-Chain Fatty Acids (MCFA), mainly lauric acid $(12: 0)^{[2]}$. The claim for its use in obesity treatment is associated with the MCFA effects such as increasing energy expenditure and fat oxidation, also reducing waist circumference $^{[2,3]}$.

Obesity is a growing global health problem aggravated by its related complications with various morbidities such as diabetes, hypertension, cardiovascular disease and cancer. According to the estimates of the World Health Organization, in 2014 more than one-third (39\%) of adults in the world aged $\geq$ 18 years were overweight and $11 \%$ of men and $15 \%$ of women worldwide were obese ${ }^{[4]}$. Furthermore, obesity associates with a low-grade inflammatory response and is often accompanied by elevated inflammatory markers such as pro-inflammatory cytokines $^{[5,6]}$.

Although the etiology of obesity is complex, diet is an important modified factor involved in obesity-induced inflammation. In addition, nutrient physiology and immunology are inextricably linked. Studies suggest that fasting or high-fat meals can induce acute episodes of nutrition-related immune activation $^{[7,8]}$. In this sense, fatty acids influence many events and cellular mechanisms, playing an important role in regulating inflammatory and immune responses ${ }^{[9]}$.

Study on animal model (C57BL/6J mice) demonstrated an association with coconut oil consumption with a down regulated production of pro-inflammatory cytokines (IL-6, IFN- $\gamma$ ) and reduced intestinal inflammation ${ }^{[10]}$. However, studies evaluating similar effect in human are scarce and controversial ${ }^{[11-13]}$.

(7) Copyrights: (C) 2016 Usuda Prado Rocha, D.M. This is an Open access article distributed under the terms of Creative Commons Attribution 4.0 International License. 
Thereby, we intend to contribute to the knowledge about the cytokines concentration, as biomarkers on inflammatory status, associated with the coconut oil consumption.

This preliminary study aimed to assess the postprandial acute effect of coconut oil intake on serum concentration of IL-2, IL-4, IL-6, IL-10, IL-17A, IFN- $\gamma$ and TNF cytokines in overweight women.

\section{Materials and Methods}

\section{Participants}

Volunteers were recruited by advertising on university campus and local radio. Eligibility criteria included healthy women (aged 20 - 45 years), overweight/obesity $\left(\geq 25 \mathrm{~kg} / \mathrm{m}^{2}\right)$, and high body fat content (>30\%) assessed by tetra polar bioelectrical impedance method (In Body®, model Y230); weight stability $(<3 \mathrm{~kg})$ and physical activity in the last 3 months. Smokers; pregnant or lactating women; subjects with chronic and inflammatory diseases; using drugs could affect the study results; consuming caffeine $\geq 1250 \mathrm{mg} /$ day and intake of one alcohol dose $(10-12 \mathrm{~g})$ per day were not included in the study. All participants were advised to maintain the level of physical activity and diet style throughout the duration of the study.

The study protocol was in accordance with to the Helsinki Declaration and it was approved by the Human Ethical Committee in Scientific Research (protocol number 541.836) of Universidade Federal de Viçosa, Brazil. Written informed consent was obtained from all the participants.

\section{Study design}

This pilot study was a clinical trial with a randomized, controlled, crossover design on the postprandial effect of two is ocaloric meals with fatty acids derived from coconut oil or olive oil on serum inflammatory cytokines. The study was conducted at the Laboratory of Energy Metabolism and Body Composition (LAMECC), Department of Nutrition and Health, Universidade Federal de Viçosa, Brazil. After an overnight fast of at least 12 h, 17 volunteers were recruited to reported to the laboratory and randomly consumed one of the test meals within $10-15 \mathrm{~min}$ of the first (baseline) blood sampling. Blood samples were then taken sequentially at 60,120 , and $180 \mathrm{~min}$ after the test meal was consumed (Figure 1). During the postprandial assessment, participants refrained from any strenuous activity and abstained from consuming any food, except water. The consumption of each test meal was separated by a washout period of at least one week between sessions.

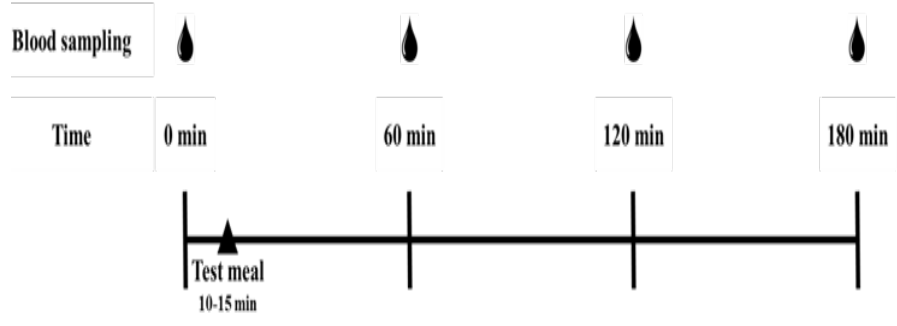

Figure 1: Experimental design of the study. Venous blood samples were collected in the fasting state ( 0 minute) and 60, 120 and 180 minutes after the start of meal consumption (10 - 15minutes).

\section{Meal composition}

The isocaloric meals ( $494 \mathrm{kcal})$ provided $45.7 \%$ of energy as fat, $39.7 \%$ as carbohydrate and $14.6 \%$ as proteins. The test meal main source of fat was the coconut oil, rich in medium chain saturated fatty acids. The control meal containing olive oil was rich in long-chain mono unsaturated fatty acids. The meals comprised $50 \mathrm{~g}$ of white bread added with $25 \mathrm{~mL}$ of the selected lipid source (coconut oil or olive oil), and 300 $\mathrm{mL}$ of water-reconstituted powdered milk ( $40 \mathrm{~g})$ added with flavoring strawberry powder $(2.7 \mathrm{~g})$, constituting 30 to $40 \%$ of the volunteers baseline estimated energy requirements. During storage, coconut oil and olive oil were kept refrigerated $\left(4^{\circ} \mathrm{C}\right)$ to avoid physicochemical changes. Nutritional composition of the meals was determined according to manufacturer's information and the total caloric value was obtained from the sum of the calories corresponding to macronutrients and was expressed in kilocalories (kcal) (Table 1).

Table 1: Macronutrient composition and energy content of coconut oil test meal and olive oil meal (control).

\begin{tabular}{|l|l|l|}
\hline Composition & Coconutoilmeal & $\begin{array}{l}\text { Olive oilmeal } \\
\text { (control) }\end{array}$ \\
\hline Calories & $494.0 \mathrm{kcal}$ & $494.0 \mathrm{kcal}$ \\
\hline Carbohydrates & $49.0 \mathrm{~g}(39.7 \% \mathrm{E})$ & $49.0 \mathrm{~g}(39.7 \% \mathrm{E})$ \\
\hline Protein & $18.0 \mathrm{~g}(14.6 \% \mathrm{E})$ & $18.0 \mathrm{~g}(14.6 \% \mathrm{E})$ \\
\hline Fat & $25.1 \mathrm{~g}(45.7 \% \mathrm{E})$ & $25.1 \mathrm{~g}(45.7 \% \mathrm{E})$ \\
\hline Saturatedfat & $23.6 \mathrm{~g}(43.0 \% \mathrm{E})$ & $3.3 \mathrm{~g}(6.0 \% \mathrm{E})$ \\
\hline Monounsaturatedfat & $1.3 \mathrm{~g}(2.4 \% \mathrm{E})$ & $20.0 \mathrm{~g}(36.4 \% \mathrm{E})$ \\
\hline Polyunsaturatedfat & $0.3 \mathrm{~g}(0.5 \% \mathrm{E})$ & $1.8 \mathrm{~g}(3.3 \% \mathrm{E})$ \\
\hline
\end{tabular}

$\% \mathrm{E}$ (\% of energy intake)

\section{Blood samples}

Blood samples were extracted after $12 \mathrm{~h}$ of fast, through intravenous puncture in the median a tecubital vein using a vacuum system, with subsequent centrifugation at $3500 \mathrm{rpm}$ for 15 $\min \left(4^{\circ} \mathrm{C}\right)$. Serum samples were stored at $-80^{\circ} \mathrm{C}$ until analysis.

Serum glucose, triglycerides and total cholesterol concentrations were determined by colorimetric enzymatic tests (Cobas Mira Plus, Roche Diagnostic Systems). The insulin concentrations were measured by electrochemilumine scence immunoassay (Elecsys-Modular-E-170, Roche Diagnostics Systems). HDL-cholesterol (HDL-c) concentrations were determined in the supernatant of serum samples following precipitation with phosphotungstic acid/ $\mathrm{Mg}^{2+}$. LDL-cholesterol (LDL-c) was calculated using the Friedewald formula.

Flow cytometry analysis was performed by using a BD FACSV erse ${ }^{\mathrm{TM}}$ flow cytometer (BD Biosciences). Serum concentrations of the cytokines interleukin-2 (IL-2), interleukin-4 (IL4), interleukin-6 (IL-6), interleukin-10 (IL-10), interleukin-17A (IL-17A), interferon gamma (IFN- $\gamma$ ) and tumour necrosis factor (TNF) were measured using commercial kit (Cytometric Bead Array CBA Human Th1/Th2/Th17 Kit, BD Biosciences) according to the manufacturers' instructions. Acquired data were analyzed using FCAP Array ${ }^{\mathrm{TM}}$ Software v3.0 (BD Biosciences) 


\section{Statistical analysis}

The cytokines area under the curve (AUC) and incremental area under the curve (iAUC) was calculated for cytokines from 0 to 3 hours, using the trapezoid rule in Graph Pad Prism 5 software (Graph Pad, La Jolla, CA, USA). Shapiro-Wilk's normality test was used to check for the normal distribution of data. Data are expressed as mean \pm SEM or median ( $225-\mathrm{p} 75^{\text {th }}$ percentiles values), when appropriate. Differences in serum cytokine concentrations of the two isoenergetic meals between time points for each test meal, and differences in cytokines concentrations between meals (coconut - olive oil) in each time point were assessed with Friedman's, followed by pair wise comparisons with a Bonferroni correction for multiple comparisons. Changes $\Delta$ (final - baseline) in cytokines concentrations and differences in the cytokines AUC and iAUC between the two isoenergetic meals were analyzed Wilcoxon signed-rank test. Statistical analysis was conducted using SPSS 20 for Windows (SPSS, Inc., Chicago, IL, USA). The criterion for statistical significance was $\alpha \leq 0.05$ for all data analyses.

\section{Results}

Seventeen women were randomly assigned to the trial, mean age at baseline of $26.69 \pm 1.12$ years, $27.50 \pm 0.32 \mathrm{~kg} / \mathrm{m}^{2}$ and $37.44 \pm 0.54 \%$ body fat (Table 2 ). Due to personal reasons, 15 women completed test (coconut oil) and 16 control (olive oil) interventions. A total of 14 participants completed both interventions. Data from all participants that completed the study were included in baseline analyses as well as in the baseline postprandial analyses of inflammatory biomarkers.
Table 2: Baseline characteristics of study participants.

\begin{tabular}{|l|c|}
\hline Characteristics & $\mathbf{n}=\mathbf{1 7}$ \\
\hline Age (years) & $26.69 \pm 1.12$ \\
\hline Body weight (kg) & $70.56 \pm 0.92$ \\
\hline Height (m) & $1.60 \pm 0.01$ \\
\hline Waist circumference (cm) & $95.09 \pm 0.97$ \\
\hline BMI (kg/m $)$ & $27.50 \pm 0.32$ \\
\hline Body fat (\%) & $37.44 \pm 0.54$ \\
\hline Cholesterol (mg/dL) & $157.83 \pm 4.93$ \\
\hline HDL-c (mg/dL) & $48.17 \pm 2.17$ \\
\hline VLDL-c (mg/dL) & $21.15 \pm 1.57$ \\
\hline LDL-c (mg/dL) & $88.51 \pm 3.94$ \\
\hline Triglycerides (mg/dL) & $105.77 \pm 7.83$ \\
\hline Glucose (mg/dL) & $81.20 \pm 1.42$ \\
\hline Insulin (uU/dL) & $10.40 \pm 1.19$ \\
\hline
\end{tabular}

Data shown as mean $\pm \mathrm{SEM}$.

Measurements of Th1, Th2 and Th17 serum cytokines (Tables 3 and 4) and delta cytokines revealed no significant differences in relation to the effect of the coconut or olive oil meals, between time points for each test meal, or differences in cytokines concentrations between meals (coconut - olive oil) in each time point. Also, no differences were observed in changes $\Delta$ (final - baseline) in cytokines concentrations, cytokines AUC or iAUC between the two tested meals.

Table 3: Postprandial effect of acute consumption of meal containing coconut oil on serum cytokines concentration $(n=15)$.

\begin{tabular}{|l|l|l|l|l|}
\hline \multirow{2}{*}{ Cytokine* $(\mathbf{p g} / \mathbf{m L})$} & \multicolumn{4}{|c|}{ Time } \\
\cline { 2 - 5 } & \multicolumn{1}{|c|}{$\mathbf{0 h}$} & \multicolumn{1}{|c|}{$\mathbf{1 h}$} & \multicolumn{1}{c|}{$\mathbf{2 h}$} & $\mathbf{3 h}$ \\
\hline IL-6 & $12.08(5.51-18.58)$ & $5.84(0.00-13.67)$ & $4.59(1.99-14.51)$ & $4.09(0.00-14.90)$ \\
\hline IL-10 & $5.26(2.26-8.37)$ & $4.03(2.78-6.05)$ & $3.93(0.83-6.07)$ & $2.83(0.00-7.51)$ \\
\hline IF-17A & $230.88(141.59-292.41)$ & $191.62(167.10-247.33)$ & $186.94(97.81-217.85)$ & $145.91(0.00-177.65)$ \\
\hline TNF & $52.52(21.80-81.76)$ & $44.29(21.80-66.30)$ & $37.96(16.47-53.27)$ & $11.345(0.00-46.05)$ \\
\hline
\end{tabular}

Data shown as median $\left(25-75^{\text {th }}\right.$ percentiles values $)$

* Values above the limit detection for $>50 \%$ samples for both IL-2 and IL-4 cytokines.

Table 4: Postprandial effect of acute consumption of meal containing olive oil on serum cytokines concentration $(\mathrm{n}=16)$.

\begin{tabular}{|l|l|l|l|l|}
\hline \multirow{2}{*}{ Cytokines(pg/mL) } & \multicolumn{4}{|c|}{ Time } \\
\cline { 2 - 5 } & \multicolumn{1}{|c|}{$\mathbf{0 h}$} & \multicolumn{1}{|c|}{$\mathbf{1 h}$} & \multicolumn{1}{c|}{$\mathbf{2 h}$} & \multicolumn{1}{|c|}{$\mathbf{3 h}$} \\
\hline IL-6 & $4.43(0.00-10.34)$ & $4.93(3.83-12.24)$ & $4.93(2.60-11.50)$ & $5.59(0.00-14.75)$ \\
\hline IL-10 & $5.50(1.85-6.62)$ & $5.18(3.02-8.54)$ & $3.87(1.85-7.25)$ & $3.87(0.85-7.40)$ \\
\hline IL-17A & $178.82(111.14-257.97)$ & $188.02(126.95-259.41)$ & $128.11(80.45-196.05)$ & $137.19(12.85-224.13)$ \\
\hline IFN & $32.93(18.65-63.62)$ & $50.58(25.69-70.47)$ & $39.14(24.31-52.52)$ & $41.93(18.50-60.48)$ \\
\hline
\end{tabular}

Data shown as median $\left(25-75^{\text {th }}\right.$ percentiles values $)$

* Values above the limit detection for $>50 \%$ samples for both IL-2 and IL-4 cytokines. 


\section{Discussion}

The media highlights coconut oil as a food with positive effects on health. Women are a target group of these marketing strategies due to their increasing demand for products that promote thermogenic effects and reduce weight and waist circumference, as claimed for coconut oil ${ }^{[12]}$. Other health claims also include its antioxidant and antimicrobial and anti-inflammatory properties ${ }^{[14-16]}$.

In our study, the intake of $25 \mathrm{~mL}$ ( $46 \%$ of meal energy) of coconut oil or olive oil (control) did not change post prandialserum anti and pro-inflammatory cytokines. Similarly, previous study evaluating the coconut oil and olive oil consumption ( $20 \%$ of daily value) during 5 weeks also verified no change on plasma inflammatory cytokines (IL-1 $\beta$, IL-6, IL-8, IFN- $\gamma$, TNF- $\alpha)^{[12]}$. Myhrstad et al. (2011) also did not achieve significant results testing meals containing $29 \mathrm{~g}$ of coconut oil on postprandial plasma pro-inflammatory cytokines (IL-6 and IL-8) or their gene expression on PBMC, at 3 and $6 \mathrm{~h}$ after consumption of the test meals. Moreover, Kontogianni et al. (2013) assessed inflammatory markers related to olive oil intake $(15 \mathrm{~mL})$ during 6 weeks and found no significant changes in inflammatory markers, including hs-PCR and TNF- $\alpha$, confirming our results. We know that saturated fats are linked to inflammatory processes ${ }^{[18]}$. However, coconut oil does not seem to have this effect, despite being source of saturated fat. In addition, coconut oil does not affect the lipemia ${ }^{[2]}$. Thus, coconut oil could be an interesting substitute for saturated fat from animal sources, possibly related to the presence of other elements including bioactive compounds.

Nevertheless, studies indicate the beneficial effect of olive oil consumption on pro-inflammatory cytokines ${ }^{[19,20]}$. PREDIMED study (PREvención con DIeta MEDiterránea), provided weekly 1 liter of olive oil for home consumption ad libitum in the context of the Mediterranean diet. After 1-year intervention, subjects with high cardiometabolic risk showed significant reduction in plasma concentrations of IL-6 and TNF receptors (TNFR60 and TNFR80) ${ }^{[19]}$. Also, the acute consumption of extra virgin olive oil $(50 \mathrm{~mL})$ reduced the postprandial concentration of TNF- $\alpha$ after $3 \mathrm{~h}$, showing an anti-inflammatory effect associated with its use ${ }^{[20]}$.

First, we emphasize that the amount used in the studies cited are far superior to that used in our study, suggesting a possible anti-inflammatory dose-response relationship regard to olive oil consumption. However, we cannot state that higher doses of coconut oil would also promote similar effect. In fact, Pietraszek et al. (2011) in a postprandial acute study found an induced pro-inflammatory response for several inflammatory genes in adipose and muscle tissue associated with high dose of coconut oil $(80 \mathrm{~g})$ consumed by healthy subjects or at risk for diabetes. In addition, in this intervention, pro-inflammatory cytokine IL-6 plasma concentrations increased in response to coconut oil consumption. Furthermore, quantities greater than applied in our research does not reflect the usual fat intake of the population studied by us.

The strengths of our study include its design (controlled, crossover clinical trial), good compliance with test meals, and the number of both pro and anti-inflammatory cytokines evaluated. However, the postprandial period of evaluation $(3 \mathrm{~h})$ may have been insufficient to detect changes in the evaluated inflammatory markers. In fact, the secretion of such proteins involves transcriptional and translational processes, which does not occur simultaneously, also, other regulatory mechanisms may be involved $^{[21]}$. This way, it would be interesting to conduct long-term feeding trials using coconut oil to evaluate the effect on the concentration of inflammatory cytokines and thereby ascertain the impact of their use for the inflammatory state, and consequently on the health of consumers.

\section{Conclusion}

Despite being a saturated fat source, the acute consumption of coconut oil did not alter the inflammatory response in healthy adult overweight women, suggesting that the consumption of moderate amounts of coconut oil $(25 \mathrm{~mL})$ does not promote the increase of postprandial inflammatory response. Further studies are needed considering a larger intervention period to check long-term effect.

Acknowledgment: The authors are grateful to all those who participated in the laboratory carrying out the work, especially to Flávia GalvãoCândido and Flávia Xavier Valente. They also thank the Fignelli Comp. Imp. Exp. LTDA who donated the coconut oil for the study.

Conflict of Interests: The authors declare no conflict of interest.

Funding and Authorship: No funding is declared. JB conceived and designed the study and was responsible for subject recruitment and data collection. DMUPR, APSC, OGLC, ALGD, LMRL, LLO and JB drafted the manuscript, participated in data analysis and interpretation. All authors critically reviewed the manuscript and approved the final version submitted for publication.

\section{References}

1. Cunningham, E. Is There Science to Support Claims for Coconut Oil? (2011) J Am Diet Assoc 111(5): 786.

2. Assunção, M.L., Ferreira, H.S., Dos Santos, A.F., et al. Effects of dietary coconut oil on the biochemical and anthropometric profiles of women presenting abdominal obesity. (2009) Lipids 44(7): 593-601.

3. St-Onge, M-P., Bourque, C., Jones, P.J.H., et al. Medium- versus long-chain triglycerides for 27 days increases fat oxidation and energy expenditure without resulting in changes in body composition in overweight women. (2003) Int J Obes Relat Metab Disord 27(1): 95-102.

4. WHO. Global status report on noncommunicable diseases. 2014.

5. Carraro, J.C.C., Hermsdorff, H.H., Puchau, B., et al. Interleukin-6 is a better metabolic biomarker than interleukin-18 in young healthy adults. (2015) J Physiol Biochem 71(3): 527-535.

6. Pourshahidi, L.K., Wallace, J.M.W., Mulhern, M.S., et al. Indices of adiposity as predictors of cardiometabolic risk and inflammation in young adults. (2015) J Hum Nutr Diet 29(1): 26-37.

7. Herieka, M., Erridge, C. High-fat meal induced postprandial inflammation. (2014) Mol Nutr Food Res 58(1): 136-146.

8. de Vries, M.A., Klop, B., Eskes, S.A., et al. The postprandial situation as a pro-inflammatory condition. (2014) Clin Investig en Arterioscler 26(4): 184-192.

9. Teng, K-T., Chang, C-Y., Chang, L.F., et al. Modulation of obesity-induced inflammation by dietary fats: mechanisms and clinical evidence. (2014) Nutr J 13: 12.

10. Mañé, J., Pedrosa, E., Lorén, V., et al. Partial replacement of dietary (n-6) fatty acids with medium-chain triglycerides decreases the incidence of spontaneous colitis in interleukin-10-deficient mice. (2009) J 
Nutr 139(3): 603-610.

11. Pietraszek, A., Gregersen, S., Hermansen, K. Acute effects of dietary fat on inflammatory markers and gene expression in first-degree relatives of type 2 diabetes patients. (2011) Rev Diabet Stud 8(4): 477-489. 12. Voon, P.T., Ng, T.K., Lee, V.K., et al. Diets high in palmitic acid (16:0), lauric and myristic acids $(12: 0+14: 0)$, or oleic acid (18:1) do not alter postprandial or fasting plasma homocysteine and inflammatory markers in healthy Malaysian adults. (2011) Am J Clin Nutr 94(6): 1451-1457.

13. Myhrstad, M.C., Narverud, I., Telle-Hansen, V.H., et al. Effect of the fat composition of a single high-fat meal on inflammatory markers in healthy young women. (2011) Br J Nutr 106(12): 1826-1835.

14. Alves, N.F., Porpino, S.K., Monteiro, M.M., et al. Coconut oil supplementation and physical exercise improves baroreflex sensitivity and oxidative stress in hypertensive rats. (2015) Appl Physiol Nutr Metab 40(4): 393-400.

15. DebMandal, M., Mandal, S. Coconut (Cocos nucifera L.: Arecaceae): In health promotion and disease prevention. (2011) Asian Pac J Trop Med 4(3): 241-247.
16. Gao, M., Singh, A., Macri, K., et al. Antioxidant components of naturally-occurring oils exhibit marked anti-inflammatory activity in epithelial cells of the human upper respiratory system. (2011) Respir Res 12(1): 92.

17. Kontogianni, M.D., Vlassopoulos, A., Gatzieva, A., et al. Flaxseed oil does not affect inflammatory markers and lipid profile compared to olive oil, in young, healthy, normal weight adults. (2013) Metabolism 62(5): 686-93.

18. Rocha, D.M., Caldas, A.P., Oliveira, L.L., et al. Saturated fatty acids trigger TLR4-mediated inflammatory response. (2016) Atherosclerosis 244: 211-215.

19. Urpi-Sarda, M., Casas, R., Chiva-Blanch, G., et al. The Mediterranean diet pattern and its main components are associated with lower plasma concentrations of tumor necrosis factor receptor 60 in patients at high risk for cardiovascular disease. (2012) J Nutr 142(6): 1019-1025. 20. Papageorgiou, N., Tousoulis, D., Psaltopoulou, T., et al. Divergent anti-inflammatory effects of different oil acute consumption on healthy individuals. (2011) Eur J Clin Nutr 65: 514-519.

21. Berg, J., Tymoczko, J., Stryer, L. Eukaryotic Transcription and Translation Are Separated in Space and Time. (2002) Biochemistry.
Online ISSN: 2377-0619

Journal Title: International Journal Food and Nutritional Science Journal Short Name: Int J Food Nutr Sci
Ommega Online Publishers

E-mail: foodscience@ommegaonline.org

Website: www.ommegaonline.org 